\title{
VORSCHLÄGE ZUR ERWEITERUNG DER SLOWENISCHEN VARIANTE DER NATÜRLICHKEITSTHEORIE
}

\section{Einleitung}

Im Aufsatz werden Vorschläge zur Erweiterung des in Slowenien entstandenen Modells der Natürlichkeitstheorie gemacht. Im weiteren Verlauf des Aufsatzes verwende ich die Akronyme NT für die Natürlichkeitstheorie in der Ausprägung ihrer bekanntesten Vertreter (Dressler, Mayerthaler, Wurzel) und SNT für das slowenische Modell zur Erweiterung der Natürlichkeitstheorie, dessen Grundidee wohl mit Orešnik (1986) erstmals international veröffentlicht wurde und seitdem in einer Reihe von Arbeiten überprüft und weiterentwickelt wird (mit Orešnik \& Teržan \& Trobevšek \& Snedec 1990 als wichtigem Meilenstein).

\section{NT 1981 und NT 1987}

Die NT gemäß Dressler \& Mayerthaler \& Panagl \& Wurzel 1987 geht von folgender Bewertung der Natürlichkeit einer sprachlichen Kategorie aus:

(1) Eine sprachliche Kategorie ist weniger matuirlich (markierter), falls sie (Schmid 1997: 340):

(a) nicht uniform enkodiert (universales Prinzip),

(b) nicht morphotaktisch und/oder morphosemantisch transparent (universales Prinzip),

(c) nicht ikonisch / diagrammatisch sind (universales Prinzip),

(d) nicht typangemessen ist (typologisches Prinzip),

(e) nicht mit den charakteristischen Struktureigenschaften des betreffenden Sprachsystems übereinstimmen (einzelsprachliches Prinzip)

(f) oder zu einer weniger stabilen oder weniger umfangreichen Flexionsklasse gehören (einzelsprachliches Prinzip).

Die NT in dieser Ausprägung wurde vor allem auf phonologische und morphologische Kategorien angewandt. In späteren Arbeiten (z.B. Dressler 1989, Dotter 1990, Schmid 1997, Mayerthaler \& Fliedl \& Winkler 1998 u.a.) wurde die Natürlichkeitstheorie auch auf andere Ebenen der Sprachbeschreibung (z.B. Textlinguistik, Syntax, Zweitspracherwerb) ausgeweitet.

In der NT 1981 und NT 1987 werden die systemunabhängigen Prinzipien der Transparenz, Uniformität und des konstruktionellen Ikonismus (Diagrammatismus) als auch der Begriff der Natürlichkeit (bzw. das Gegenstück: Markiertheit) verwendet. Diese Prinzipien spielen auch in der SNT 1990 und SNT 1999 eine grundlegende Rolle zur Bewertung der Natürlichkeit: 
(2) Ein sprachliches Element ist gemäß Mayerthaler (1987: 49) maximal transparent, wenn es sich nach dem Fregeschen Prinzip der Kompositionalität richtet (morphosemantische Transparenz) und wenn Silben- und Morphemgrenzen übereinstimmen (morphotaktische Transparenz).

(3) Die Symbolisierung bzw. Enkodierung einer sprachlichen Form ist gemäß Mayerthaler (1981: 34) maximal uniform, wenn sie eineindeutig ist (d.h. wenn einer Funktion genau eine Form entspricht und umgekehrt) und wenn sie allomorphiefrei ist. Ist eine sprachliche Form nur allomorphiefrei, jedoch polyfunktional, liegt schwache Uniformität vor.

(4) Wenn eine semantisch markiertere Kategorie merkmalhaft kodiert wird, ist sie gemäß Mayerthaler (1987: 48-49) konstruktionell ikonisch, sonst nicht-ikonisch oder sogar kontraikonisch.

(5) Mit Hinblick auf die universalgrammatischen Präferenzen ist eine sprachliche Form gemäß Mayerthaler (1987: 49) maximal matürlich (maximal ummarkiert), wenn sie den Prinzipien der Transparenz, Uniformität und Ikonizität entspricht. Mayerthaler (1981: 46) legt folgende Prinzipienhierarchie fest: konstruktioneller Ikonismus > (»vor« bzw. »über«) uniformer Symbolisierung > Transparenz. Prinzipien, die hierarchisch untergeordneten Bereichen angehören, sind im Falle eines Natuirlichkeitskonflikts die Verlierer (Mayerthaler 1981: 46).

\section{SNT 1990}

Orešnik \& Teržan \& Trobevšek \& Snedec (1990: 5) postulieren die Existenz von verstärkten und nicht-verstärkten Konstruktionen einerseits (bei synchronischem Bezug auch starke und schwache Varianten genannt) sowie geschwächten und nichtgeschwächten andererseits:

(6) Verstärkte Konstruktionen sind ausdrucksseitig aufwendiger, semantisch eindeutiger, nicht so sprechereffizient, jedoch hörerfreundlicher als die entsprechenden nicht-verstärkten Konstruktionen. Eine verstärkte Konstruktion erleichtert somit die Dekodierung der vom Textproduzenten intendierten Bedeutung. Für die geschwächten bzw. nicht-geschwächten Konstruktionen gilt das Umgekehrte.

Die Grundannahme von Orešnik \& Teržan \& Trobevšek \& Snedec (1990: 6) besagt folgendes:

(7) Verstärkte Konstruktionen behaupten sich im Zeitraum, in dem sie als Variante einer entsprechenden nicht-verstärkten Konstruktion auftreten, vorzugsweise in relativ komplexen grammatischen Umgebungen, können sich jedoch in einem späteren Stadium auch in anderen Umgebungen verbreiten. Für die sogenannten geschwächten Konstruktionen soll die umgekehrte Aussage gelten.

Im Rahmen der SNT 1990 sind wohl bislang die meisten Monographien entstanden, z.B. Teržan 1988, Teržan 1991, Trobevšek 1987, Trobevšek 1991, Snedec 1988, Petrič 1990, Petrič 1995a, Marinčić 1990. Die Grundannahmen der SNT 1990 wurden auch im Rahmen zahlreicher Konferenzbeiträge und wissenschaftlicher Zeit schriftenartikel geprüft und die Ergebnisse international bekannt gemacht. Nicht 
zuletzt haben wohl auch die beiden internationalen Natürlichkeitssymposien in Maribor 1993 und 1996 (Boretzky \& Dressler \& Orešnik \& Teržan \& Wurzel 1995; Teržan 1998) zur Profilierung des in Slowenien entstandenen linguistischen Ansatzes beigetragen.

\section{SNT 1999}

Die SNT 1990 und die SNT 1999 stellen eine auf die Syntax angewandte Erweiterung der systemunabhängigen NT dar (vgl. Mayerthaler 1981 und Dressler, Mayerthaler, Panagl, Wurzel 1987).

Gemäß Orešnik (1999: 192) wird die Aussage in (7) präzisiert, denn es wird behauptet, dass für die Variante eines Variantenpaars und ihre Umgebung die folgenden altermativen Eigenschaften gelten:

(8) Alternativen für eine Variante und ihre Umgebung (nach Orešnik 1999: 172 - 175)

\begin{tabular}{|c|c|c|c|c|c|}
\hline \multicolumn{2}{|c|}{ Variante: } & verbindet sich mit & $\begin{array}{c}\text { Merkmal: } \\
\text { sym }\end{array}$ & Verknüpfung & $\begin{array}{c}\text { Merkmal: } \\
\text { sem }\end{array}$ \\
\hline sym & + & $\rightarrow$ & + & und / oder & - \\
\hline sym & - & $\rightarrow$ & - & und / oder & + \\
\hline sem & + & $\rightarrow$ & - & und / oder & + \\
\hline sem & - & $\rightarrow$ & + & und /oder & - \\
\hline \multicolumn{5}{r|}{} \\
\end{tabular}

[+sym]: natürlicher hinsichtlich Enkodierung (Transparenz, Uniformität, Ikonizität); [-sym]: weniger natürlich hinsichtlich Enkodierung (Transparenz, Uniformität, Ikonizität); [+sem]: natürlicher hinsichtlich semantischer Komplexität; [-sem]: weniger natuirlich hinsichtlich semantischer Komplexität Anmerkung: statt der sonst üblichen Größer-und Kleiner-Symbole $(<>$ ) verwende ich m.E. visuell leichter interpretierbare Plus- und Minuszeichen

(9) Zumindest ein [+sym]-Wert zeigt die Tendenz, sich zumindest mit einem zusätzlichen [+sym]-Wert und/oder mit zumindest einem [-sem]-Wert zu verbinden;

(10) Zumindest ein [-sym]-Wert zeigt die Tendenz, sich zumindest mit einem zusätzlichen [-sym]-Wert und/oder mit zumindest einem [+sem]-Wert zu verbinden;

(11) Zumindest ein [+sem]-Wert zeigt die Tendenz, sich zumindest mit einem zusätzlichen [+sem]-Wert und/oder mit zumindest einem [-sym]-Wert zu verbinden;

(12) Zumindest ein [-sem]-Wert zeigt die Tendenz, sich zumindest mit einem zusätzlichen [-sem]-Wert und/oder mit zumindest einem [+sym]-Wert zu verbinden.

Der metasprachliche Ausdruck »sich mit ... verbinden» bezieht sich sowohl auf die beobachtete sprachliche Einheit als auch auf ihre unmittelbare Umgebung. 
Im Rahmen der SNT 1999 sind mittlerweile mehrere wissenschaftliche Bücher (Orešnik 1999, Orešnik 2001, Dobrovoljc 2005) und eine Reihe von Zeitschriften- und Konferenzbeiträgen veröffentlicht worden.

\section{Fragestellungen zur Erweiterung der SNT}

Als Ansatz, eine Erweiterung der SNT vorzuschlagen, soll die Beschreibung der Grundannahmen in (8) bis (12) aus Orešnik (1999: 172 - 175) sowie die Beschreibung der Sem- und Sym-Skalen in Dobrovoljc (2005: 28) dienen.

Dobrovoljc (2005: 26 - 30) beschreibt, wie die die Sem- und Sym-Skalen aus Orešnik (1999) und Orešnik (2001) aufeinander bezogen werden. Ein Fall ist die folgende Sem-Skala (Dobrovoljc 2005):

(13) >sem (weniger transparent, transparenter) / syntaktische Einheit

Im Hinblick auf die kognitive Komplexität sind weniger transparente syntaktische Einheiten natürlicher als transparentere. - Im Interesse des Sprechers ist es, weniger umfangreiche syntaktische Einheiten zu äußern, weil ihm dies weniger Mühe bereitet.

Setzt man voraus, dass diese Annahme stimmt, erhebt sich die Frage, welche Faktoren bewirken, dass der Sprecher in wirklichen Textzusammenhängen statt der ausdrucksökonomischeren syntaktischen Einheit eine aufwendigere (also etwa eine transparentere) präferiert?

Eine weitere Fragestellung betrifft einzelsprachliche Unterschiede. In Orešnik (1999), Orešnik (2001) und Dobrovoljc (2005) werden verschiedene grammatische Paradigmen behandelt und hinsichtlich ihrer Natürlichkeit bewertet. Die Natürlichkeitsbewertung ist in vielen Fällen nur auf bestimmte Sprachen beziehbar. Welche Faktoren bewirken nun, dass in einer Sprache beispielsweise eine weniger transparente Konstruktionsweise geläufiger ist als in einer anderen?

\section{Zur Rangfolge der SNT-Prinzipien in Texten}

Sprachliche Konstruktionen sind das Produkt von Optimalisierungsprozessen in der Kommunikation, bei möglichst geringen kognitiven Kosten soll eine möglichst große Wirkung erreicht werden (Minimax-Prinzip). Zwischen den sprachlichen Bedürfnissen des Textproduzenten und des Textrezipienten besteht ein Konflikt: Geringer Kodieraufwand hat einen höheren Dekodieraufwand zur Folge. Die in Texten verwendeten sprachlichen Konstruktionen stellen mehr oder weniger erfolgreiche Kompromisslösungen zur Bewältigung des kommunikativen Konflikts dar.

Die vom Textproduzenten intendierte Bedeutung (d.h. der propositionelle Gehalt und/oder der illokutive Inhalt einer Äußerung bzw. eines Textsegments bzw. eines Textes) kann vom Textrezipienten besser verstanden werden, wenn sie transparent, uniform und ikonisch übermittelt wird, außerdem wenn sie mit typangemessenen und/oder systemangemessenen Mitteln kodiert wird (d.h. mit prototypischen sprachlichen Mitteln, häufiger verwendeten Ausdrücken, ...) übermittelt werden.

Der kognitive Aufwand beim Kodieren und Dekodieren kann oft nur indirekt bewertet werden. Dazu können folgende heuristische Hilfsmittel verwendet werden (vgl. auch Mayerthaler 1981, Orešnik (2003: 59- 60), Dobrovoljc (2005: 32 - 33, 36, 38): 
1. das phylogenetische Alter (Sprachgeschichte)

2. das ontogenetische Alter (Spracherwerb)

3. Sprachpathologie

4. Versprecher

5. Prototypizität

6. Strukturprinzipien (Symmetrie, Integration)

7. Gebrauchsfrequenz

8. Sprachtypologie (Universalien: Verbreitung in den Sprachen der Welt)

9. Paradigmengröße (einzelsprachliche Musterbildung)

10. Spezieller Gebrauch (Irregularität)

11. Kategoriengebrauch und Prozessdurchführung (Transformationen)

12. Textualitätskriterien (Kohärenz, Kohäsion, Situationalität, Akzeptabilität, ...)

13. $\ldots$

Die Art und Weise der sprachlichen Kodierung und Dekodierung ist von vor- und außersprachlichen Umständen abhängig. Ausgangspunkte für den Textproduzenten:

1. Bewertung des außersprachlichen Situationszusammenhangs und der Teilnehmer

2. vorsprachliche Zielsetzung (Illokution, Sprechhandlung)

3. intendierter propositioneller Gehalt

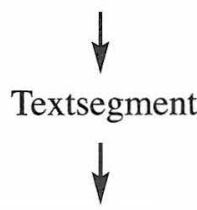

Ausgangspunkte für den Textrezipienten:

4. Bewertung des außersprachlichen Situationszusammenhangs und der Teilnehmer

5. Erschließung des vorsprachlichen Ziels des Sprechers (Illokution, Sprechhandlung)

6. Erschließung des intendierten propositionellen Gehalts

Der außersprachliche Situationszusammenhang und die Teilnehmer können im Rahmen eines Textvorkommens meist als Konstanten betrachtet werden.

Ziel der folgenden Darstellung ist eine Einschätzung der Kodiervor- und -nachteile von sprachlichen Konstruktionen. Zu diesem Zweck wird eine Tabelle erstellt, in der ein Vorteil einer Konstruktion auf einer bestimmten Werteskala durch einen höheren Rang ausgedrückt wird.

Als Input in einer solchen Tabelle können folgende Größen gewählt werden:

(a) der vom Sprecher intendierte propositionelle Gehalt - prop

(b) der vom Sprecher intendierte illokutive (modale) Inhalt - ill

(c) der vom Sprecher geäußerte sprachliche Ausdruck - con

Als Output einer solchen Tabelle können folgende Größen gewählt werden:

(a) theoretisch mögliche sprachliche Konstruktionen oder Varianten - con 
(b) die vom Hörer verstandene propositionelle Bedeutung - prop

(c) die vom Hörer verstandene illokutive (modale) Bedeutung - ill

In der folgenden Darstellung wird die Prädikation P (behandel-, operier-, ...) mit den dazugehörigen semantischen Rollen Agens (Arzt, ...) und Patiens (Patient, ...) als Input in die Bewertungstabelle eingesetzt. Im Output der Tabelle erscheinen verschiedene theoretisch mögliche Konstruktionen, deren Kodiervorteile und -nachteile im Folgenden auf verschiedenen Natürlichkeitsskalen bewertet werden sollen. Die Darstellung bezieht sich auf die deutsche Sprache, kann aber zumindest teilweise auch für andere Sprachen, insbesondere Nominativ-Akkusativ-Sprachen, Gültigkeit haben.

\begin{tabular}{|l|l|}
\hline Input & Output \\
\hline Assertion: & (A) Hauptsatz (Aussagesatz, Aktivsatz), z.B. Der Arzt behandelt den Patienten \\
\cline { 2 - 2 } & (B) Hauptsatz (Aussagesatz, Passivsatz), z.B. Der Patient wird (vom Arzt) behandelt \\
\hline (Agens, & (C) Nebensatz (Aktivsatz), z.B. dass der Arzt den Patienten behandelt \\
\hline Patiens) & (D) Nebensatz (Passivsatz), z.B. dass der Patient (vom Arzt) behandelt wird \\
\cline { 2 - 2 } & (E) Infinitivsatz, z.B. den Patienten zu behandeln \\
\cline { 2 - 2 } & (F) Nominalisierung, z.B. die Behandlung (des Patienten) (durch den Arzt) \\
\hline
\end{tabular}

1. Ausgehend vom konstruktionellen Ikonismus (k. Ikon.: ein komplexerer Inhalt korreliert mit einer komplexeren Form) sind (B), (D) und (F) bei fehlendem Agens (bzw. fehlendem Agens und Patiens) weniger ikonisch als (A) und (C), da die zuerst genannten drei Konstruktionen das Prädikat zweiteilig realisieren, die Konstruktion (E) hingegen immer kontraikonisch (aufgrund obligatorisch nicht realisiertem Agens). Daher erhalten (B), (D) und (F) Rang 2, (A) und (C) Rang 1, die Konstruktion (E) dagegen Rang 3.

2. Eine Sprechhandlung (hier eine Behauptung (Assertion)) wird durch einen Satz eindeutiger ausgedrückt als durch eine Nominalphrase, d.h. die illokutive Transparenz (ill. Tra.) von (A) und (B) ist größer als von (C), (D), (E) und (F). Daher erhalten (A) und (B) auf dieser Skala Rang 1 (den höchsten Rang), (C) und (D) Rang 2, (E) und (F) Rang 3.

3. Ausgehend vom Kodieraufwand (Ökon) ist eine Konstruktion für den Textproduzenten einfacher, wenn sie fakultative Bestandteile enthält bzw. wenn sie bestimmte Bestandteile einer Proposition nicht enthält. (A) und (C) enthalten nur obligatorische Bestandteile, (B), (D) und (E) enthalten eine fakultative Konstituente bzw. erlauben die Nicht-Realisierung einer Konstituente (nämlich des Agens), die Konstruktion (F) ermöglicht sogar die Aussparung von zwei Bestandteilen (nämlich des Agens und des Patiens). Daher erhält (F) Rang 1, (B), (D) und (E) Rang 2, die Konstruktionen (A) und (C) dagegen Rang 3.

4. Rektionsrichtung (Rekt.): Im Deutschen kann die Konstituentenstellung SOV als zugrunde liegend angesehen werden, d.h. Links-Rektion. In den Konstruktionen (B), (C), (D) und (E) regiert das Hauptverb nach links, daher erhalten sie Rang 1. In (A) kann das Hauptverb in Zweitstellung auftreten und dann nur indirekt (über die Spur) nach links regieren, daher erhält (A) Rang 2. In $(F)$ wird nach rechts regiert, daher erhält $(F)$ nur Rang 3. 
5. Ausgehend von der anthropozentrischen und egozentrischen Perspektive sind in Nominativ-Akkusativ-Sprachen die Konstruktionen (A) und (C) typangemessener (Typ) als (B), (D), (E) und (F), denn in (A) und (C) wird die Erwartung „Agens vor Patiens“ erfüllt, in (B), (D), (E) und (F) dagegen nicht. Daher erhalten (A) und (C) auf dieser Skala Rang 1, (B), (D) und (F) Rang 2, (E) hingegen wegen obligatorisch fehlendem Agens nur Rang 3.

6. Paradligmengröße (Paradig.): Alle Prädikate können in (A), (C) und (E) eingesetzt werden, nicht jedoch in (B), (D) und (F), denn es gibt einerseits nicht passivfähige Verben, andererseits aber nicht-nominalisierbare Verben. Die Paradigmen (A), (C) und (E) sind größer als die Paradigmen (B), (D) und (F). Daher erhalten (A), (C) und (E) Rang 1, (B), (D) und (F) dagegen nur Rang 2.

7. Prototypische Funktion: Sätze sind der prototypische Ausdruck für einen propositionellen Gehalt. Prototypische Sätze enthalten ein finites Verb (Vfin). Daher erhalten die satzartigen Konstruktionen (A), (B), (C) und (D) Rang 1. (E) enthält immerhin noch eine infinite Verbform und erhält daher Rang 2. Nominalisierungen sind dagegen als Nominalphrasen der prototypische Ausdruck für Teile von Sätzen, d.h. Satzkonstituenten oder Valenzpartner von Verben. Sie enthalten keine Verbform. Daher erhalten Sie nur Rang 3.

8. Hauptsätze sind funktionell weniger eingeschränkte Konstruktionen als Nebensätze, Infinitivsätze oder Nominalisierungen. Letztere sind gewöhnlich einem anderen sprachlichen Element untergeordnet (Subord.): (C) und (D) einem Subjunktor, (E) einem Matrixverb im übergeordneten Satz, von dem aus ersichtlich ist, ob Subjekt- oder Objektkontrolle vorliegt (in manchen Fällen, insbesondere bei adverbialen Infinitivsätzen, kann auch eine Abhängigkeit von einem Subjunktor vorliegen), und (F) einem Matrixverb im Trägersatz. (A) und (B) erhalten daher Rang 1, die übrigen Konstruktionen Rang 2.

\begin{tabular}{|l|l|c|c|c|c|c|c|c|c|c|}
\hline Input & Output & Subord. & Vfin & Paradig. & Typ & Rekt. & Ökon & Ill. Tra. & K.Ikon. & Sum. \\
\hline Ass: & (A) HS-A & 1 & 1 & 1 & 1 & 2 & 3 & 1 & 1 & 11 \\
\cline { 2 - 12 } P \\
\cline { 2 - 11 } (Ag) HS-P & 1 & 1 & 2 & 2 & 1 & 2 & 1 & 2 & 12 \\
\cline { 2 - 11 } Pat) & (C) NS-A & 2 & 1 & 1 & 1 & 1 & 3 & 2 & 1 & 12 \\
\cline { 2 - 11 } & (D) NS-P & 2 & 1 & 2 & 2 & 1 & 2 & 2 & 2 & 14 \\
\cline { 2 - 11 } & (E) INF & 2 & 2 & 1 & 3 & 1 & 2 & 3 & 2 & 16 \\
\cline { 2 - 11 } & (F) NOM & 2 & 3 & 2 & 2 & 3 & 1 & 3 & 2 & 18 \\
\hline
\end{tabular}

Mit Hilfe dieser (hierarchisch noch nicht geordneten) acht Parameter (d.h. acht Ordinalskalen) können die sechs Konstruktionstypen hinsichtlich ihrer Natürlichkeit eingeordnet werden. Auf der linken Seite der unten abgebildeten Skala werden die Konstruktionen abgebildet, die hinsichtlich der oben verwendeten Parameter höher eingeordnet worden (d.h. die Rangsumme ist niedriger). Auf der rechten Seite der Skala sind Konstituenten mit einer höheren Rangsumme abgebildet. Je weiter links eine Konstruktion auf der unten folgenden Skala eingeordnet ist, umso natürlicher ist sie für den Ausdruck eines propositionellen Gehalts bzw. umso präferenter ist sie in Texten. 
Die Reihenfolge der oben verwendeten acht Parameter ist noch nicht hierarchisch (d.h. nach ihrer Stärke) geordnet. Gemäß den Vertretern der NT 1987 ist jedoch vorgesehen, dass einzelsprachliche Parameter (wie Systemangemessenheit oder Paradigmengröße) einen höheren Rang einnehmen als typologische oder universelle Parameter, d.h. dass einzelsprachliche Parameter auf der Grundlage universeller und typologischer Prinzipien, aber auch im Konflikt mit diesen, die Herausbildung des Sprachsystems entscheidend in bestimmte Bahnen lenken. Die Reihenfolge der acht Parameter in der obigen Tabelle richtet sich nach der Hierarchisierung der NT 1987 nur insofern, als die universellen (möglicherweise weniger einflussreichen) Prinzipien weiter rechts in der Tabelle erscheinen als typologische oder einzelsprachliche. Aufgrund der oben verwendeten Rangsummenmethode wird die Stärke der einzelnen oben verwendeten Prinzipien allerdings noch nicht berücksichtigt. Allerdings ist leicht einzusehen, dass bei hoher Prominenz des Parameters der illokutiven Transparenz (d.h. auch bekannt als Präferenz für direkte Sprechakte gegenüber indirekten) Konstruktionen wie $(\mathrm{E})$ und $(\mathrm{F})$ kaum noch in Frage kommen und Konstruktionen wie (A) und (B) an Präferenz gewinnen.

Weitere Parameter können die oben dargestellte Rangfolge der Konstruktionen (wie sie sich aus der Rangsumme ergibt) auf jeden Fall verändern (vgl. auch Petrič 1995b). Eine Konstruktion, die nach den oben verwendeten acht Parametern als weniger natürlich eingestuft wurde (z.B. ein Passivsatz), kann unter besonderen Umständen an Akzeptabilität gewinnen und in der obigen Präferenzskala weiter nach links rücken:

9. Bei Definitheitsunterschieden (Def.): Das Definitheitsprinzip ist einflussreicher als die Belebtheitshierarchie oder das Agensprinzip, das Agensprinzip dominanter als das Belebtheitsprinzip. Sind sowohl Agens als auch Patiens definit, dominiert das Prinzip »Agens vor Patiens“. Ist jedoch das Agens indefinit, das Patiens dagegen definit, kommt das Prinzip „Definites vor Indefinitem“ zur Geltung. Im Falle eines definiten Patiens und eines indefiniten Agens sind (B), (D) und (F) angemessener (Rang 1), (A) und (C) weniger angemessen (Rang 2). Auf (E) ist die Definitheitsskala (zumindest in dieser Ausprägung) nicht anwendbar. Entsprechende Definitheitsunterschiede bewirken im Deutschen, dass Passivkonstruktionen oder Nominalisierungen eine präferentere Stellung einnehmen können (in der unten verwendeten Skala durch einen Pfeil nach links abgebildet). Ein Passivsatz (B) mit definitem Patiens kann bei Verwendung der Rangsummenmethode zumindest als ebenso präferent wie ein Aktivsatz (A) eingeordnet werden. $\mathrm{Ob}$ dann im Text tatsächlich ein Passiv verwendet wird, hängt aber auch von anderen Faktoren ab. Mit Bezug auf eine Einzelsprache kann man die Stärke der einzelnen Parameter festlegen. Man könnte sich beispielsweise vorstellen, dass in einer flexionsarmen Sprache wie dem Englischen das Defi- 
nitheitsprinzip eine größere Stärke aufweist als in einer flexionsreicheren Sprache wie dem Deutschen.

$\stackrel{<+ \text { nat }}{\longleftrightarrow} \stackrel{<\text {-nat }>}{\longrightarrow}$
(A)
(C)
(D) $\leftarrow \leftarrow \leftarrow$
(E)
(F) $\leftarrow \leftarrow \leftarrow$
(B) $\leftarrow \leftarrow \leftarrow$

10. Im Text ist auch die Thema-Rhema-Gliederung (TRG; thematische Progression) bei der Reihenfolge der Satzkonstituenten $\mathrm{zu}$ berücksichtigen: Nach dem Themaprinzip ist die Reihenfolge Thema vor Rhema vorherrschend. Ist beispielsweise das Patiens (trotz Indefinitheit) thematisch und das Agens (trotz Definitheit) rhematisch, wird das Agensprinzip zugunsten des Themaprinzips zurückgestellt, d.h. der Einfluss des Agensprinzips auf die Reihenfolge der Satzkonstituenten wird zugunsten des Themaprinzips vermindert. Da im Deutschen das Subjektprinzip (Subjekt vor Objekt) einflussreicher ist als das Themaprinzip, kann beispielsweise ein Aktivsatz (A) seinen hohen Präferenzrang zugunsten eines Passivsatzes (B) verlieren. Denn im Passivsatz (B) wird sowohl das Themaprinzip (Thema vor Rhema) als auch das Subjektprinzip (Subjekt vor Objekt) berücksichtigt. Entsprechendes gilt für den Vergleich von (C) und (D). Auf (E) und (F) ist das Themaprinzip in dieser Ausprägung aus bekannten Gründen nicht anwendbar.

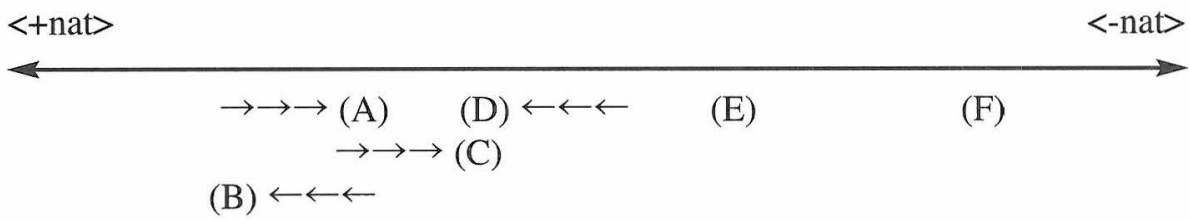

(A) Der ARZT operiert einen Patienten (nicht die Krankenschwester).

(B) Ein Patient wird vom ARZT operiert (nicht von der Krankenschwester).

11. Die Textsorte, die sich aus Globalziel, kommunikativen Umständen und Teilnehmern ergibt, beeinflusst ebenfalls den Gebrauch von sprachlichen Konstruktionen (vgl. Petrič 1998a). Anders ausgedrüickt: sprachliche Konstruktionen zeigen nicht nur eine Affinität zu bestimmten sprachlichen Umgebungen, sondern auch zu bestimmten außersprachlichen Umgebungen. Nehmen wir die Grundtextsorten (GTS) aus Diewald 1991 (vgl. auch Petrič 1998a) als Grundlage zur Bewertung der Distribution sprachlicher Konstruktionen, stellt sich heraus, dass (B), (C), (D), (E) und (F) in markierteren Grundtextsorten (insbesondere in schriftlichen Fernmonologen) häufiger auftreten als in weniger markierten Grundtextsorten (wie mündlicher Nahdialog oder mündlicher Ferndialog). 

(A)
$\rightarrow \rightarrow \rightarrow(B)$
$\rightarrow \rightarrow \rightarrow(C)$
(B) $\rightarrow \rightarrow \rightarrow(\mathrm{D})$
D) $\rightarrow \rightarrow \rightarrow$
$(\mathbb{E})$

12. Letztendlich spielen überhaupt Textualitätskriterien (Beaugrande \& Dressler 1992, Dressler 1989) wie Kohärenz, Kohäsion, Informativität, Akzeptabilität, Situationalität, Intertextualität eine Rolle, welche sprachliche Konstruktion sich unter welchen Umständen in realen Textzusammenhängen durchsetzt bzw. an Präferenz gewinnt.

Welche Konstruktion im Text tatsächlich gewählt wird, hängt davon ab, welche Rangfolge (welches Gewicht) die einzelnen (universellen, typologischen, sprachsystemspezifischen, pragmatischen) Prinzipien einnehmen (vgl. auch das Ranking in der Optimalitätstheorie).

\section{Einzelsprachliche Unterscheidung durch rangierte SNT-Prinzipien}

Die zweite Fragestellung betrifft die Rangfolge der SNT-Prinzipien (Parameter), um einzelsprachliche Unterschiede zu beschreiben und zu erklären.

\section{Ausgangspunkt meiner Darstellung}

Orešnik behandelt in seinem Artikel (Orešnik 2000) die Natürlichkeitsskalen >sem (+/-A, -A) und >sem (+/-A, +A). Die Natürlichkeitsskalen folgen wohl der allgemeinen Annahme der Markiertheitstheorie, dass das Spezielle (+A) oder (-A) markierter (weniger natürlich) ist als das Allgemeine (+/-A). Im Aufsatz möchte ich mich eingangs mit der unten angeführten Annahme und Vorhersage über englische abhängige Sätze mit finiter und infiniter Verbform aus Orešnik (2000) beschäftigen. Dabei soll der Blick danach vor allem auf meine Beurteilung der deutschen Infinitivsätze gelenkt werden.

»English. With non-finite clauses, « [-fin] »the lack of a clause link « [-CLL] »is normal« (Orešnik 2000: 239, Nr. 2). Orešniks Fortsetzung (Orešnik 2000: 239-240, Nr. 2):

Wenn

(a) $>$ sem (+fin, -fin) / clause (vgl. Mayerthaler \& Fliedl \& Winkler 1998: 325) und wenn

(b) $>$ sem (+/-CLL, -CLL)/ clause type

und wenn

(c) $[>\mathrm{sem}] \rightarrow[>\mathrm{sem}]$ und

(d) $[<$ sem $] \rightarrow[<$ sem $]$

dann

(e) $[+$ fin $] \rightarrow[+/-$ CLL $]$ in

(f) $[$-fin $] \rightarrow[-\mathrm{CLL}]$. 
Nach (a) ist ein finiter Satz hinsichtlich seiner semantischen Komplexität natürlicher als ein infiniter (Petrič 1996, vgl. Petrič 1998b). Gemäß (b) ist ein Satztyp, der ein "Clause Link" [CLL] (fakultativ) zulässt, hinsichtlich seiner semantischen Komplexität natürlicher als ein Satztyp, der ein „Clause Link“ überhaupt nicht zulässt. Nach (c) zeigt ein >sem-Parameter die Tendenz, sich mit zumindest einem anderen $>$ sem-Parameter zu verbinden. Nach (d) zeigt ein <sem-Parameter die Tendenz, sich mit zumindest einem anderen <sem-Parameter zu verbinden. Wenn (a) - (d) gilt, dann folgen daraus die beiden Tendenzen, (e) dass unter den finiten Satztypen nur relativ wenigen ein „Clause Link“ (obligatorisch) fehlt, und (f), dass unter den nicht-finiten Satztypen möglichst allen ein „Clause Link“ fehlt.

Im Deutschen kann eine derartige Verallgemeinerung auf alle abhängigen Sätze mit infiniter Verbform nicht formuliert werden, denn im Deutschen findet man neben [+fin, +/-CLL] auch Satztypen wie [-fin, +/-CLL], also nicht nur bzw. nicht vorwiegend [-fin, -CLL], wie für das Englische aus (f) folgt.

\section{Der Begriff Clause link}

Aus dem Artikel von Orešnik (2000) ist leider nicht ersichtlich, wie die Kategorie clause link [CLL] definiert ist. Deshalb möchte ich zunächst erläutern, wie ich diesen Begriff im Weiteren verwende. Die Bewertung, ob es sich um einen Satztyp mit Clause Link [+CLL] oder ohne Clause Link [-CLL] handelt, ist auf jeden Fall definitionsabhängig. Deshalb möchte ich zunächst einige Beispiele aus einer Standardgrammatik zur Illustrierung der Problematik vorführen (vgl. Helbig \& Buscha (101987: 660):

(1) Er war so klug, daß er seinen Fehler einsah / _ seinen Fehler einzusehen.

(2) Er war klug genug, daß er seinen Fehler einsah / _ seinen Fehler einzusehen.

(3) Das Wasser war so kalt, daß man nicht darin baden konnte.

(4) Das Wasser war zu kalt, als daß man darin baden konnte / könnte [T.P.].

(5) Das Wasser war zu kalt, um darin baden zu können.

(6) Wir freuen uns (darauf), _ ihn zu sehen.

Was kann noch als „Clause Link“ [+CLL] aufgefasst werden und was nicht mehr [CLL]?

[-CLL]

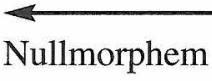

genug
[+CLL]

so

darauf

dass

Die Einordnung auf der oben angeführten Skala richtet sich nach den folgenden Kriterien: (i) der Ausdruck leitet den abhängigen Satz [+depend] ein, also [+intro]; (ii) der Ausdruck ist bedeutungsentleert [+empty]; z.B. das Präpositionaladverb darauf hat die Eigenschaften [-intro, +empty]. Es bieten sich zwei extreme Alternativen an: 
(A) Wenn adverbiale Ausdrücke wie genug, so, darauf oder die Infinitivpartikel zu als [+CLL] gewertet werden, dann haben alle oben angeführten Infinitivsätze die Eigenschaft [+CLL].

(B) Wenn lediglich der Subjunktor daß / dass als [+CLL] eingeordnet werden, dann hat nur ein Infinitivsatz in den oben angeführten Beispielen die Eigenschaft [+CLL], nämlich der in (5).

Beispiel (2) zeigt (so wie (1)) noch eine Unterscheidungsmöglichkeit: die Bedeutung des Subjunktors dass ist zu allgemein, als dass er ein konsekutives Verhältnis [+consec] im Satzgefüge anzeigen könnte. Dies wird erst durch das Adverb genug ermöglicht. Demnach wäre es angemessen, auch das Adverb genug als »clause link « einzuordnen. Der Unterschied zwischen den Sätzen [+fin] und [-fin] in (2) läge demnach darin, dass das „Clause Link“ im ersten Satzyp [+fin] zweiteilig wäre (d.h. genug + dass), im zweiten Satztyp [-fin] dagegen nur einteilig (nämlich nur aus genug bestehend). Die Kombination des Präpositionaladverbs darauf mit dem Subjunktor dass könnte demnach als prototypischeres zweiteiliges „Clause Link“" bezeichnet werden, die Kombination des quantitaven Adverbs genug mit dem Subjunktor dass dagegen als weniger prototypisches "Clause Link“, so trägt doch die erst genannte Kombination die Eigenschaften [-intro, +empty und +intro, +empty], die zuletzt genannte Kombination dagegen die Eigenschaften [-intro, -empty und +intro, +empty].

Deshalb liegt die m.E. angemessenste Grenze zwischen den Kategorienwerten [+CLL] und [-CLL] gerade zwischen dem Adverb so [-empty] und dem Präpositionaladverb darauf [+empty]. Im Weiteren werde ich angesichts meiner Fragestellung, ob deutsche Infinitivsatztypen mehrheitlich die Eigenschaft [+CLL] aufweisen, dennoch die ungünstigere Definition des Begriffs „Clause Link“" wählen, d.h. (B), wonach dem syntaktischen Kriterium Genüge geleistet wird, dass der Ausdruck in der Rolle eines „Clause Link“ den abhängigen Satz einleitet und damit nicht Bestandteil des übergeordneten Satzes ist.

\section{Deutsche Neben- und Infinitivsätze mit und ohne ,Clause Link ${ }^{66}$}

Wenn wir nun die Beschreibung der Infinitivsätze [+inf] in einer deutschen Standardgrammatik berücksichtigen, z.B. die in Helbig \& Buscha ( ${ }^{10} 1987$ : 656-661), dann sind im Deutschen sowohl abhängige Sätze mit den Merkmalen [+fin, +/-CLL] als auch mit den Merkmalen [-fin, +/-CLL] zu finden, also im Gegensatz zum Englischen, wie aus der Schlussfolgerung (f) in Orešnik (2000) folgt, nicht nur bzw. nicht vorwiegend Infinitivsätze mit den Merkmalen [-fin, -CLL].

Wenn ein deutscher Infinitivsatz in der Funktion einer Adverbialbestimmung [+adv] auftritt, weist er meist das Merkmal [+CLL] auf, erscheint ein deutscher Infinitivsatz dagegen als Subjekt oder Objekt [-adv], hat er gewöhnlich die Eigenschaft [-CLL].

Die meisten Adverbialbestimmungen sind nicht valenzgebunden [-val], während das Subjekt und alle Objekte valenzgebunden [+val] sind. Als Ausnahmen unter den Adverbialbestimmungen dürfen z.B. Richtungsbestimmungen, sogenannte Direktivergänzungen, gelten, denn sie weisen die Merkmale [+CLL] und [+val] auf. 
Allerdings erscheinen die Direktivergänzungen meines Wissens nach nicht in der Form von Infinitivsätzen.

Hier folgen einige Beispiele aus Helbig \& Buscha ( ${ }^{10} 1987$ : 656ff.) zur Illustration, und zwar zunächst Infinitivsätze mit dem Merkmal [+val], wobei ein Infinitivsatz in (7) als Präpositionalobjekt, in (8) als Subjekt, in (9) als Akkusativobjekt, in (10) als Genitivobjekt und in (11) als Präpositionalobjekt zum Substantiv Recht auftritt:

(7) Wir freuen uns, ihn zu sehen. [-fin, +inf, +val, +prp, -CLL]

(8) Ihn zu treffen ist mir peinlich. [-fin, +inf, +val, +sub, -CLL]

(9) Sie erlaubte ihm, früher nach Hause zu gehen. [-fin, +inf, +val, +akk, -CLL]

(10) Peter ist sich bewußt, seinen Freund verletzt zu haben. [-fin, +inf, +val, +gen, CLL]

(11) Er hat das Recht, diese Papiere zu lesen. [-fin, +inf, +val, +prp N, -CLL]

Noch einige Beispiele aus Helbig \& Buscha (101987: 656ff.) zur Illustration, und zwar mit nicht-valenzgebundenen adverbialen Infinitivsätzen mit den Merkmalen [+adv] [-val]:

(12) Er geht in das Bad, (an)statt zu arbeiten. [-fin, +inf, -val, +substitutiv, +CLL]

(13) Er geht in die Bibliothek, um dort zu arbeiten. [-fin, +inf, -val, +final, +CLL]

(14) Er geht in den Betrieb, ohne dort zu arbeiten. [-fin, +inf, -val, +kommitativ, $+\mathrm{CLL}]$

Die valenzgebundenen Satzglieder betrachte ich als natürlicher, die nicht-valenzgebundenen Satzglieder hingegen als weniger natürlich. Dies möchte ich damit begründen, dass der Textrezipient valenzgebundene Satzglieder eher erwartet als nicht-valenzgebundene Satzglieder, d.h. die zuerst genannten Satzglieder redundanter sind als die zuletzt genannten:

(g) >sym (+erwartet, -erwartet)/Redundanz

(h) $>$ sym ( +val, -val) / Erwartbarkeit der Satzglieder

Ähnlich (wenn auch nicht gleich) wird von Orešnik (2000: 246) zwischen »subject or object gap« einerseits und »adverbial or prepositional object gap « andererseits unterschieden, indem erläutert wird, dass »subject and objects are easier for the hearer to process than adverbial or prepositional object gaps.«:

(i) $>$ sym (subject/object gap, adverbial/prepositional object gap) / inf. clause in English.

Aus den Natürlichkeitsbewertungen (a) - (d), (g) in (h) folgt, dass wir unter den deutschen Satztypen mit den Merkmalen [+fin, +val] häufiger solche mit dem Merkmal [+/CLL] finden können als unter den Satztypen mit den Merkmalen [-fin, -val]. 
Hier folgen einige Beispiele aus der deskriptiven Grammatik Helbig \& Buscha (101987: 653, 660) zur Illustration, und zwar zuerst Satztypen mit den Merkmalen [+depend, +fin, +val, -adv]:

(15) Ich dachte, er hätte seine Prüfung abgelegt / daß er seine Prüfung abgelegt hätte.

(16) Es ist besser, du kommst pünktlich / daß du pünktlich kommst.

Die valenzgebundenen finiten Nebensätze [+fin, +val], die als Subjekt [+sub] oder Objekt $[+$ akk, +prp,$\ldots]$ auftreten, erlauben also die Eigenschaft [+/-CLL], die wenigen valenzgebundenen finiten Nebensatztypen in Adverbialfunktion [+fin, +val, +adv] erlauben dagegen nur das Merkmal [+CLL], z.B. die Richtungsbestimmungen (Direktivergänzungen). Das Kriterium [+/-adv] ist demnach im Deutschen einflussreicher als das Merkmal [+/-val].

Sowohl finite abhängige Sätze in Subjekt- oder Objektfunktion, d.h. solche mit den Merkmalen [+depend, +fin, +val, -adv], als auch finite abhängige Sätze in Adverbialfunktion, d.h. solche mit den Merkmalen [+depend, +fin, +/-val, +adv], können entweder das Merkmal [+CLL] oder (in wenigeren Fällen) das Merkmal [-CLL] aufweisen (siehe Beispiele (17) - (19) unten).

Hier folgen wiederum einige Beispiele aus der deskriptiven Grammatik Helbig \& Buscha $\left({ }^{10} 1987: 653,660\right)$ mit den Satztypen [+depend, +fin, -val, +adv], die uns zeigen, dass es sich lediglich um drei Fälle von valenzungebundenen finiten Nebensätzen [+fin, -val] handelt, und zwar um Konzessivsätze [+conces], um Konditionalsätze [+cond] und Konsekutivsätze [+consec], die das Merkmal [+/-CLL] erlauben, während die meisten anderen Nebensatztypen mit den Merkmalen [+depend, +fin, -val, +adv], die im Deutschen wesentlich zahlreicher sind, lediglich das Merkmal [+CLL] zulassen:

(17) Mag er auch noch wenig Erfahrung haben / Obwohl er noch wenig Erfahrung hat, so weiß er doch sehr viel.

(18) Solltest du sie treffen / Falls du sie treffen solltest, sage ihr bitte Bescheid.

(19) Er war klug genug, seinen Fehler einzusehen / daß er seinen Fehler einsah.

Im Deutschen bestimmt die grundlegende Abgrenzung zwischen infiniten Satztypen mit den Merkmalen [+adv] und [-adv] auch im Wesentlichen die Grenze zwischen den inifiniten Satztypen mit den Merkmalen [+CLL] und [-CLL]. Das Valenzmerkmal [+/-val] leistet dazu im Deutschen keinen entscheidenden Beitrag, so dass man in der Natürlichkeitsskala (h) das Merkmal [+/-val] durch [+/-adv] ersetzen sollte.

Im Deutschen folgen die finiten und infiniten abhängigen Sätze der Natürlichkeitsskala >sem (+/-A, +A) (vgl. dazu Orešnik 2000: 253ff.), denn mehrere deutsche finite Nebensätze [+fin] erlauben das Merkmal [+/-CLL], deutsche Infinitivsätze [+inf] dagegen relativ häufiger [+CLL]. Deutsche Infinitivsätze mit dem Merkmal [+adv] gehören fast ausschließlich zum Typ [+CLL], was darauf deutet, dass 
sie im Rahmen der Natürlichkeitsskala >sem $(+/-\mathrm{A},+\mathrm{A})$ und nicht gemäß der Natürlichkeitsskala $>$ sem $(+/-$ A, - A) betrachtet werden können - im Unterschied zu den englischen Infinitivsätzen.

\section{Zusammenfassung des 7. Abschnitts}

Zurück zu der Grundannahme in Orešnik (2000: 239, Nr. 2): In englischen Satzkonstruktionen mit infiniter Verbform [-fin] fehlt gewöhnlich ein „Clause Link“ (,Konjunktionswort"), also [-fin] [-CLL].

Im Deutschen kann diese Schlussfolgerung höchstens auf Infinitivsätze in Subjektund Objektfunktion (also [+val, -adv]) übertragen werden, denn diese weisen tatsächlich die Eigenschaft [-CLL] auf, was bedeutet, dass sie als Fall der Natürlichkeitsskala $>$ sem $(+/-\mathrm{A},-\mathrm{A})$ betrachtet werden könnten.

Infinitivsätze mit den Merkmalen [+/-val, +adv] gehören in den meisten Fällen zum Typ [+CLL], was bedeutet, dass sie als Fall der Natürlichkeitsskala >sem $(+/-\mathrm{A},+\mathrm{A})$ aufgefasst werden könnten.

Wenn man davon ausgeht, dass wesentlich weniger Objektsatztypen als verschiedene Arten von Adverbialsätzen unterschieden werden können, kann die Annahme, dass im Deutschen (so wie im Englischen) mehr Infinitivsatztypen ohne „Clause Link“ [-CLL] auftreten, nicht bestätigt werden, denn die meisten Infinitivsatztypen [-fin, +inf] weisen im Deutschen das Merkmal [+CLL] auf.

Im Deutschen kann man die Stärke der Parameter [+/-fin], [+/-val] und [+/-adv] folgendermaßen festlegen: [+/-fin] $>[+/$-adv $]>[+/$-val $]$, d.h. der einflussreichste Parameter auf das Auftreten eines "Clause Link“ in einem abhängigen Satz ist der Finitheitsparameter, gefolgt vom Adverbialparameter. Der Valenzparameter spielt im Vergleich zu diesen beiden eine untergeordnete Rolle. In deutschen Infinitivsätzen spielt somit der Adverbialparameter eine entscheidende Rolle für das Auftreten eines „Clause Link" („Konjunktionswortes"). In einer anderen Sprache, z.B. dem Englischen, scheint der Adverbialparameter diesbezüglich eine geringere Rolle zu spielen als im Deutschen. 


\section{Literatur}

DE BEAUGRANDE, Robert Alain \& Dressler, Wolfgang Ulrich (1992): Uvod v besediloslovje. Založba Park Ljubljana. BORETZKY, Norbert \& Dressler, Wolfgang \& Orešnik, Janez \& Teržan, Karmen \& Wurzel, Wolfgang (1995)

(Hgg.): Natürlichkeitstheorie und Sprachwandel / Teorija naravnosti in jezikovno spreminjanje. Beiträge zum internationalen Symposium über "Natürlichkeitstheorie und Sprachwandel" an der Universität Maribor vom 13.5.-15.5.1993. Bochum: Brockmeyer.

Diewald, Gabriele (1991): Deixis und Textsorten im Deutschen. Niemeyer: Tübingen.

DOBRovolIC, Helena (2005): Slovenska teorija jezikovne naravnosti. Ljubljana: Založba ZRC.

DOTTER, Franz (1990): Nichtarbitrarität und Ikonizität in der Syntax. Hamburg: Buske.

DRESSLER, Wolfgang Ulrich (1989): Semiotische Parameter einer textlinguistischen Natürlichkeitstheorie. Wien: Verlag der Österreichischen Akademie der Wissenschaften.

DRESSLER, Wolfgang Ulrich \& Mayerthaler, Willi \& Panagl, Oswald \& Wurzel, Wolfgang Ullrich, (eds.) (1987): Leitmotifs in Natural Morphology. Amsterdam: Benjamins.

Helbig \& Buscha $\left({ }^{10} 1987\right)$ : Deutsche Grammatik. Ein Handbuch für den Ausländerunterricht. Leipzig: Verlag Enzyklopädie.

MARINčIć, Branka (1990): Zapletenost oziralnih odvisnikov v delih Thomasa Elyota. <Komplexität der Relativsätze in den Werken von Thomas Elyot>. Magistrsko delo <Magisterarbeit>. Filozofska fakulteta $<$ Philosophische Fakultät>. Ljubljana.

MAYERTHALER, Willi (1981): Morphologische Natürlichkeit. Wiesbaden: Athenaion.

MAYERTHALER, Willi (1987): System-independent morphological naturalness. In: Dressler, W.U., Mayerthaler, W., Panagl, O., Wurzel, W.U. (eds.) 1987, 25-58.

MAYERTHALER, Willi \& Fliedl, Günther \& Winkler, Christian (1998): Lexikon der natürlichkeitstheoretischen Syntax und Morphosyntax. Tübingen: Stauffenburg.

OREŠNIK, Janez (1986): The Obligatorium of unemphatic pronoun subjects in Germanic languages. In: Filologija 14, 261-270. Zagreb.

OREšNIK, Janez \& Snedec, Andrej \& Teržan, Karmen \& Trobevšek-Drobnak, Frančiška (1990): Introduction to the Subsequent Three Papers in the Present Volume. In: Linguistica XXX, 5-12. Ljubljana.

OREŠNIK, Janez (1999): Krepke in šibke dvojnice v skladnji. Strong and weak variants in syntax. Ljubljana: SAZU.

OREŠNIK, Janez (2000): The scale formats >sem (+/-A, -A) and >sem (+/-A, +A). In: Linguistica XL/2, 237-262. Ljubljana.

OREŠNIK, Janez (2001): A predictable aspect of (morpho)syntactic variants. Predvidljiv vidik (obliko)skladenjskih dvojnic, Ljubljana: SAZU.

OREŠNIK, Janez (2003): Naturalness. Some Norwegian (morpho)syntactic examples. In: Norsk Lingvistik Tidsskrift 21, $57-70$.

PETRIč, Teodor (1990): Posamostaljenja v knjižni nemščini <Nominalisierungen im Standarddeutschen>. Magistrsko delo <Magisterarbeit $>$. Filozofska fakulteta <Philosophische Fakultät $>$. Ljubljana.

PETRIč, Teodor (1995a): Naklonsi členki v nemščini. <Modalpartikeln im Deutschen>. Doktorska disertacija $<$ Dissertation $>$. Filozofska fakulteta $<$ Philosophische Fakultät $>$. Ljubljana.

PETRIč (1995b): Modalpartikeln und Natürlichkeitstheorie. In: Boretzky, N. \& Dressler, W. \& Orešnik, J.\& Teržan, K. \& Wurzel, W. (1995) (Hgg.). 277 - 292.

PETRIč, Teodor (1996): Odgovori na pripombe prof. Orešnika. Workshop-Manuskript.

PETRIč, Teodor (1998a): Korrelationen zwischen merkmalhaften oder merkmallosen syntaktischen Varianten und verschiedenen Graden der Textsortenkomplexität. In: Linguistica XXXVIII, 1. 201 - 224.

PETRIč (1998b): Zu geschwächten Konstruktionen am Beispiel deutscher Nominalisierungen mit abstraktem Verbalnomen als Kopf. In: Teržan-Kopecky (1998) (ur., Hg.), 133 - 158.

ScHMiD, Stephan. 1997. The Naturalness Differential Hypothesis: Cross-linguistic Influence and Universal Preferences in Interlanguage Phonology and Morphology. Folia Linguistica XXXI/3-4, 331-348.

SNEDEC, Andrej (1988): Potek skladenjske spremembe. Angleški glagol do <Syntaktischer Wandel. Das englische Verb $d o>$. Magistrsko delo <Magisterarbeit $>$. Filozofska fakulteta $<$ Philosophische Fakultä $>$. Ljubljana.

TERžAN (1988): Trpnik kot skladenjski pojav v nemščini <Das Passiv als syntaktische Erscheinung im Deutschen>. Magistrsko delo <Magisterarbeit>. Filozofska fakulteta <Philosophische Fakultät>. Ljubljana.

TERŽAN-KOPECKY (1991): Prihodnjik kot skladenjska okrepitev v nemščini - diahroni in sinhroni vidiki <Das Futur als syntaktische Verstärkung im Deutschen - diachronische und synchronische Aspekte>. Doktorska disertacija <Dissertation>. Filozofska fakulteta <Philosophische Fakultät>. Ljubljana.

TERŽAN-KOPECKY (1998) (Hg.): Zbornik referatov II. mednarodnega simpozija o teoriji naravnosti 23. do 25. maja 1996. Sammelband des II. internationalen Symposions zur Natürlichkeitstheorie. Maribor: Pedagoška fakulteta, Pädagogische Fakultät.

Trobevšek DrobnaK, Frančiška (1987): Potek skladenjske spremembe. Angleški razširjeni časi <Syntaktischer Wandel. Die englischen Expanded Tenses>. Magistrsko delo <Magisterarbeit>. Filozofska fakulteta $<$ Philosophische Fakultä $\triangleright$. Ljubljana. 
TROBEV̌̌EK-DROBNAK, Frančiška (1991): Skladnja staroangleških glagolov s predpono ge- v primerjavi s skladnjo staroangleških glagolov brez predpone <Syntax der altenglischen Verben mit dem Präfix ge. im Vergleich zur Syntax der altenglischen Verben ohne Präfix $>$. Doktorska disertacija $<$ Dissertation $>$. Filozofska fakulteta $<$ Philosophische Fakultät $>$. Ljubljana.

\section{Povzetek \\ PREDLOGI ZA RAZŠIRITEV SLOVENSKE RAZLIČICE TEORIJE NARAVNOSTI}

Jezikovne zgradbe so rezultat optimalizacije v procesu sporočanja: tvorec besedila želi s čim manj kognitivnega napora doseči čim večji učinek (načelo minimax). Med jezikovnimi težnjami tvorca besedila in sprejemnika besedila prihaja do nasprotij: varčno izražanje otežuje razumevanje in obratno. Jezikovne zgradbe, ki nastopajo v besedilih, predstavljajo več ali manj uspešne kompromise za rešitev komunikativnih nasprotij. Sprejemnik besedila lažje razume pomen povedi (tj. stavčno vsebino in/ali namen povedi), če jo tvorec besedila posreduje $\mathrm{z}$ manj zaznamovanimi ali naravnejšimi (razvidnejšimi, ikoničnejšimi, tipsko ustreznejšimi, prototipičnejšimi, ...) jezikovnimi sredstvi. V sestavku avtor razglablja o možnostih za razširitev slovenske teorije naravnosti: kateri dejavniki povzročajo, da se npr, tvorec besedila odloči za potratnejšo, vendar razvidnejšo jezikovno zgradbo, in kako bi z razporejanjem načel naravnosti mogli prikazovati naravnostne razlike jezikovnih zgradb znotraj enega jezika in naravnostne razlike jezikovnih zgradb različnih jezikov. 